\title{
Der IGQ - Ein Messinstrument für die Wirksamkeit von Gamification
}

\author{
Romina Kettner, Katja Herrmanny, Werner Gaulke \\ Universität Duisburg-Essen
}

\section{Zusammenfassung}

Leistungs- und Effektivitätssteigerung, stärkere Unternehmensbindung sowie erhöhte MitarbeiterInnenzufriedenheit zählen zu den wesentlichen Motivatoren für den Einsatz von spielerisch angereicherten Prozessen in Unternehmen. Die anhaltende Diskussion über die Wirksamkeit solcher Gamification-Anwendungen kann unter anderem auf das Fehlen geeigneter Messinstrumente insbesondere für die „,weichen“ Faktoren zurückgeführt werden. Um diese Lücke zu schließen und eine Basis für die empirisch fundierte Wirksamkeitsbeurteilung und Vergleichbarkeit von internalen Gamification-Anwendungen zu schaffen, wurde der Internal Gamification Questionnaire (IGQ) entwickelt. Konstruktionsprozess und Validierung des aus neun Subskalen bestehenden Fragebogens werden in diesem Beitrag vorgestellt.

\section{$1 \quad$ Einleitung}

Eine höhere Unternehmensbindung in Form von Loyalität, gesteigerte Effektivität und Zufriedenheit gehören zu den angestrebten Zielen von Gamification im Unternehmenseinsatz (Rauch 2013; Hamari 2013). Dabei kann Gamification sowohl an die KundenInnen als auch die MitarbeiterInnen eines Unternehmens gerichtet sein. Erstere werden z.B. in Form von Marketingaktionen angesprochen während letztere durch interne Prozesse und Anwendungen erreichbar sind.

Beide Fälle eint, dass bisher keine ausreichend evaluierten Messinstrumente zur Wirksamkeitskontrolle eingesetzter Gamification-Elemente existieren (Hamari 2013; Cechanowicz et al. 2013). Diesen Umstand bezeichnet Maan (2013) als „Lack of 'Win States' or `Success Metrics`“, wonach das gewünschte Verhalten und die Erfolgsmaße, welche über die Güte einer Gamification-Anwendung entscheiden, nicht klar definiert sind. Um eine qualifizierte Aussage darüber treffen zu können, ob sich z.B. die Motivation durch die Verwendung von Gamification erhöht, sind objektive und transparente Messinstrumente nötig. Der vorgestellte Fragebogen ist speziell auf die Evaluation von Anwendungen im Sinne der 
sogenannten internalen Gamification (Werbach \& Hunter 2012), die Optimierung interner Geschäftsprozesse, zugeschnitten, wodurch eine innerbetriebliche Nutzung in Unternehmen vorgesehen ist. In diesem Beitrag wird ein solches Messinstrument in Form des Internal Gamification Questionnaire (IGQ) vorgestellt.

\subsection{Stand der existierenden Messinstrumente}

Nach bisherigen Erkenntnissen ist sich die Wissenschaft nicht einig, inwiefern GamificationAnwendungen einen effektiven Nutzen bringen (Iosup \& Epema 2014). Die positiven Effekte, die Gamification-Anwendungen häufig nachgesagt werden, sind gemäß Hamari (2013) empirisch oftmals nur unzureichend untersucht. Während einige empirische Arbeiten den positiven Einfluss von Gamification-Anwendungen auf motivationale Affordances nachgewiesen haben wollen, berichten andere, dass die Lang-Zeit-Effektivität ausbliebe (Farzan et al. 2008a; Farzan et al. 2008b; Hamari 2013). Als ein Grund für diese heterogenen Forschungsergebnisse kann das Fehlen geeigneter Messinstrumente angesehen werden (Hamari et al. 2014; Cechanowicz et al. 2013). Betrachtet man die Evaluationsmöglichkeiten für die Wirkungskomponenten von Gamification, so fällt das Defizit an geeigneten Instrumenten auf (Mitgutsch \& Alvarado 2012; Cechanowicz et al. 2013). Denn nicht nur der messbare Nutzen von Gamification ist kaum erforscht, sondern ebenfalls die Auswirkungen auf einzelne Facetten wie beispielsweise Gameful-Experience (Nacke et al. 2010), UserExperience (Ullrich \& Diefenbach 2010) oder auch das Flow-Erleben, gemäß Csikszentmihalyi (1991), in Spielsituationen (Nacke et al. 2009). Zusammenfassend lässt sich festhalten, dass derzeit keine zweckdienlichen Messinstrumente bekannt sind, welche eine geeignete Evaluationsmöglichkeit für internale Gamification darstellen. Der im Folgenden vorgestellte Fragebogen ermöglicht nicht nur die Vergleichbarkeit von GamificationAnwendungen (Hamari et al. 2014), sondern bietet ein Instrument um deren Wirksamkeit zu messen.

\section{Konstruktion des IGQ}

Grundlegend für die Beurteilung der Wirksamkeit von Gamification-Anwendungen ist die Ermittlung geeigneter messbarer Faktoren. Einer davon ist die User-Experience, deren Analyse laut Cechanowicz et al. (2013) Implikationen für Gamification-Designansätze beinhaltet. Jung et al. (2010) führen ergänzend zahlreiche Beispiele für Studien an, die sich mit Gameful-Experience und der erlebten Zufriedenheit von Nutzenden in diesem Zusammenhang beschäftigen, sodass die Kategorie Erlebnis den Einfluss von Gamification abbildet. Im Unternehmenskontext, spielt die soziale Interaktion häufig in Form von kollaborativen Gruppensettings eine wichtige Rolle und dient in vielen Studien als Wirksamkeitsfaktor (Jung et al. 2010). Darüber hinaus ist das Verhältnis zwischen den Unternehmensangehörigen und ihrer Organisation ein wesentlicher Punkt, da der Unternehmenserfolg auch von der Loyalität, dem Commitment und der Identifikation seitens der MitarbeiterInnen mit dem Unternehmen abhängt (Borzaga \& Tortia 2006; Van Knippenberg \& Sleebos 2006). In unmittelbarem Zusammenhang mit diesen Merkmalen steht 
ebenso die Compliance, welche durch sogenannte Ethic Programs Einzug in die vornehmlich US-amerikanische Arbeitswelt erhielt (Weaver \& Trevino 1999) und die Triade der bindungsrelevanten Faktoren vollendet. Anknüpfend an diese Betrachtungen erarbeitete Schering (2015) u.a. die Kategorien Verhalten, Bindung und Erleben, welche einschließlich ihrer Wirkungsfaktoren im Rahmen der Fragebogenentwicklung zu betrachten sind und insgesamt neun separate Subskalen ergeben.

\begin{tabular}{l|l} 
Kategorien & Faktoren/Subskalen \\
\hline Verhalten & Soziale Interaktion \\
Bindung & Loyalität, Commitment, Compliance \\
Erleben & Flow, Zufriedenheit, Engagement, User Experience, Gameful Experience
\end{tabular}

Tabelle 1 - Faktoren des IGQ mit den jeweiligen Subskalen.

Die Itemkonstruktion erfolgte für jede dieser Subskalen mittels der deduktiven und der induktiven Strategie. Demnach wurden theoretisch fundierte Verhaltensindikatoren in Form von Aussagen formuliert und die daraus resultierenden Items durch die inferenzstatistische Methode der exploratorischen Faktorenanalyse überprüft. Infolge der Recherche und Sichtung des theoretischen Fundaments und der Identifikation von Kernelementen, welche die einzelnen Konstrukte dezidiert beschreiben, entstand ein initialer Itempool von 101 Aussagen. Aus diesem wurde nach einer abschließenden inhaltlichen Analyse, die sich auf die Kongruenz zwischen Item und zu messendem Konstrukt (Bühner 2011) fokussierte, die 85 geeignetsten Items für die Überprüfung im Rahmen des Pretests ausgewählt. Anhand von 25 qualitativen Interviews (17 männlich, 8 weiblich; $M=32$ Jahre) nach der Methode der explorativen Befragung wurden mögliche Schwächen der Itemkonstruktion und der Verständlichkeit des Inhalts identifiziert (Raab-Steiner \& Benesch 2010). Das Sprachverständnis der Probanden wurde erfasst um ungeeignete Probanden auszufiltern. Für 56 Items ergab sich Modifizierungsbedarf, 26 Items wurden direkt in die Zweitfassung übernommen und drei Items erwiesen sich als ungeeignet weshalb sie eliminiert wurden.

\section{Evaluation des IGQ}

Zur Überprüfung des Messinstruments erfolgte eine empirische Evaluation. Demnach sollte die Korrelation des Vergleichsinstruments, bestehend aus vorhandenen konstrukterfassenden Fragebögen, mit den konstruierten Subskalen des IGQ berechnet werden, um Aussagen über die Qualität des Letzteren hinsichtlich der konvergenten und divergenten Validität zu treffen. Gegenstand der Hauptstudie war die 82 Items umfassende Zweifassung des IGQ. Bevor diese in Form einer Online-Befragung beantwortet werden sollten, wählten die 91 Befragten (31 männlich, 60 weiblich; $M=25.5$ Jahre) eine von acht Gamification-Anwendungen aus, die sie in einem definierten Zeitraum nutzten. Die Zuteilung zu einem der beiden Fragebögen wurde randomisiert, wonach sich 46 Testpersonen in der Kontrollgruppe und 45 in der Experimentalgruppe wiederfanden. 


\subsection{Ergebnisse}

Die Evaluation in der Hauptstudie diente der Überprüfung der internen Validität, welche für alle neun Subskalen durch eine Hauptkomponentenanalyse mit anschließender Rotation (Promax) sichergestellt werden konnte. Zudem stellte die Itemanalyse einen wesentlichen Bestandteil der methodischen Überlegungen dar. So wurde die Itemtrennschärfe mit der Produkt-Moment-Korrelation ermittelt und alle Items mit einem Trennschärfekoeffizienten von unter .40 entfernt. Darüber hinaus wurde die Itemschwierigkeit bestimmt, wodurch sich ein durchschnittlicher Schwierigkeitsindex von 58,97 berichten lässt. In diesem Zusammenhang verhalf die Schätzung der internen Konsistenz durch die Berechnung des Reliabilitätskoeffizienten Cronbachs Alpha zu der Ermittlung der finalen Items des IGQ. Schließlich ließ sich die externe Validität mittels einer Korrelationsanalyse hinsichtlich der konstruierten Items des IGQ und existierenden Items der Vergleichsinstrumente verifizieren. Was die externe Validität anging, konnte ansatzweise für die Subskalen Compliance und Commitment die divergente Validität nachgewiesen werden. So korrelierte Erstere schwach negativ $\left(\mathrm{r}_{\mathrm{s}}(91)=-.28, \mathrm{p}=.064\right)$, wenn auch knapp nicht signifikant, mit der Organizational Citizenship Behavior Scale von Moorman und Blakely (1992,1995), welche die Loyalität im Arbeitskontext erhebt. Letztere Subskala wies einen marginal signifikant gewordenen, schwach negativen Zusammenhang $\left(\mathrm{r}_{\mathrm{s}}(91)=-.29, \mathrm{p}=.056\right)$ mit dem Minnesota Satisfaction Questionnaire (Hirschfeld, 2000) zur Erfassung der arbeitsbezogenen Zufriedenheit auf. Für die Subskala Flow konnte die divergente Validität anhand der positiven Korrelation mit der negativ gepolten Mindful Attention Awareness Scale (Brown \& Ryan, 2003) $\left(\mathrm{r}_{\mathrm{s}}(91)=.32\right.$, p $<.05)$ zur Erfassung der Geistesabwesenheit nachgewiesen werden.

\begin{tabular}{|c|c|c|c|}
\hline Subskala & $\begin{array}{l}\text { Cron- } \\
\text { bachs } \alpha\end{array}$ & $\begin{array}{l}\text { Schwierig- } \\
\text { keitsindex }\end{array}$ & Vergleichsinstrumente \\
\hline $\begin{array}{l}\text { Soziale Interaktion } \\
\text { (9 Items) }\end{array}$ & .90 & 35,76 & $\begin{array}{l}\text { Dimension Scales of Social Interaction } \\
\text { (Lechler 2001) }\end{array}$ \\
\hline $\begin{array}{l}\text { Loyalität } \\
\text { (4 Items) }\end{array}$ & .87 & 63,63 & $\begin{array}{l}\text { Organizational Citizenship Behavior } \\
\text { Scale (Moorman \& Blakely 1992,1995) }\end{array}$ \\
\hline $\begin{array}{l}\text { Commitment } \\
\text { (6 Items) }\end{array}$ & .83 & 57,72 & $\begin{array}{l}\text { Organizational Commitment } \\
\text { Questionnaire (Mowday et al. 1982) }\end{array}$ \\
\hline Compliance (3 Items) & .69 & 70,55 & \\
\hline $\begin{array}{l}\text { Flow-Erleben } \\
\text { (4 Items) }\end{array}$ & .78 & 35,83 & $\begin{array}{l}\text { Flow State Scale-2 (Jackson \& Eklund } \\
\text { 2002), Mindful Attention Awareness } \\
\text { Scale (Brown \& Ryan, 2003) }\end{array}$ \\
\hline $\begin{array}{l}\text { Job-Zufriedenheit } \\
\text { (5 Items) }\end{array}$ & .82 & 65,76 & $\begin{array}{l}\text { Rev. Vers. Minnesota Satisfaction } \\
\text { Questionnaire (Hirschfeld 2000), } \\
\text { Maslach Burnout Inventory - General } \\
\text { Survey (Maslach \& Leiter 1997) }\end{array}$ \\
\hline $\begin{array}{l}\text { Engagement } \\
\text { ( } 5 \text { Items) }\end{array}$ & .87 & 58,96 & $\begin{array}{l}\text { Utrecht Work Engagement Scale } \\
\text { (Schaufeli et al. 2002), Maslach Burnout }\end{array}$ \\
\hline
\end{tabular}




\begin{tabular}{|c|c|c|c|}
\hline & & & $\begin{array}{l}\text { Inventory - General Survey (Maslach \& } \\
\text { Leiter 1997) }\end{array}$ \\
\hline $\begin{array}{l}\text { User Experience } \\
\text { (6 Items) }\end{array}$ & .93 & 53,77 & $\begin{array}{l}\text { Usability Metric for User Experience } \\
\text { (Finstad 2010) }\end{array}$ \\
\hline $\begin{array}{l}\text { Gameful Experience } \\
\text { ( } 7 \text { Items) }\end{array}$ & .86 & 64,55 & $\begin{array}{l}\text { Game Experience } \\
\text { (Ijsselsteijn et al. 2013) }\end{array}$ \\
\hline
\end{tabular}

Tabelle 2: Übersicht über die Subskalen des Internal Gamification Questionnaire

\section{$4 \quad$ Fazit}

Das vorliegende Messinstrument bietet erstmals einen umfassenden Ansatz der Evaluation und Vergleichbarkeit von Gamification-Anwendungen, was in dieser Form bisher nicht möglich war. Einer der Gründe für seine Einzigartigkeit liegt in dem modularen Aufbau des Messinstruments, wodurch es vielseitig und flexibel einsetzbar ist. Diese Qualität erweist sich insbesondere für den Einsatz in der praktischen Anwendung als vorteilhaft (Bauer und Hauptmann 1999). Durch die Berechnung eines spezifischen Summenscores für jede einzelne Subskala, können leicht die Stärken und Schwächen der betrachteten GamificationAnwendungen abgelesen werden und ebenfalls Unterschiede über spezifische MitarbeiterInnengruppen hinweg identifiziert werden. Der Praxiseinsatz wird durch die Testlänge von nur insgesamt 49 Items für alle Subskalen begünstigt, denn damit beträgt die Durchführungsdauer unter Verwendung aller Subskalen etwa 25 Minuten, wobei die Entwicklung einer Kurzversion in weiterführenden Arbeiten verfolgt werden könnte. Hinsichtlich der Überprüfung der konvergenten und divergenten Validität konnten nur für zwei der neun Subskalen erfolgreiche Ergebnisse erzielt werden, was möglicherweise auf die unzureichende Eignung der Stichprobeauswahl zurückzuführen ist. Diese bestand zu 57\% aus Studierenden, die nicht der Hauptzielgruppe des IGQ entsprechen. Im Rahmen einer zukünftigen Validierung des IGQ sollte eine Evaluation im Arbeitsalltag mit bereits länger im Unternehmen genutzten Gamification-Anwendungen erfolgen, um Realbedingungen zu schaffen. Trotz der offenen Forschungspunkte bildet der IGQ aufgrund seiner guten Reliabilität und der zufriedenstellenden internen Validität bereits jetzt einen guten Ansatz für ein umfassendes Messinstrument für Gamification. Während die erste Version des IGQ zunächst in Englisch verfasst wurde um eine bessere Gegenüberstellung mit den ebenfalls englischen Vergleichsinstrumenten zu erlangen, wird die nächste Revision auch in deutscher Sprache erscheinen.

Die in diesem Artikel diskutierte Version 1.0 des IGQ ist auf http://gamification-evaluation.de verfügbar.

\section{Literaturverzeichnis}

Borzaga C. \& Tortia, E. (2006). Worker Motivations, Job Satisfaction, and Loyalty in Public and Nonprofit Social Services. Nonprofit and voluntary sector quarterly, 35(2), 225-248. 
Brown, K. W. \& Ryan, R. M. (2003). The benefits of being present: Mindfulness and its role in psychological well-being. Journal of personality and social psychology, 84(4), 822-848.

Bühner, M. (2011). Einführung in die Test- und Fragebogenkonstruktion. 3. Auflage. München: Pearson.

Cechanowicz, J., Gutwin, C., Brownell, B., \& Goodfellow, L. (2013). Effects of gamification on participation and data quality in a real-world market research domain. In Proceedings of the First International Conference on Gameful Design, Research, and Applications. ACM, 58-65.

Csikszentmihalyi, M. (1991). Flow: The Psychology of Optimal Experience. New York: Harper Perennial.

Farzan, R., DiMicco, J., Millen, D., Brownholtz, B., Geyer, W. \& Dugan, C. (2008a). Results form Deploying a Participation Incentive Mechanisms within the Enterprise. In Proceedings of the twentysixth annual sigchi conference of human factors in computing systems. ACM Press.

Farzan, R., DiMicco, J., Millen, D., Brownholtz, B., Geyer, W. \& Dugan, C. (2008b). When the experimente is over: Deploying an incentive system to all the users. In Proceedings of the Symposium on Persuasive Technlogy. In conjunction with the AISB.

Finstad, K. (2010). The Usability Metric for User Experience. Interaction with Computers, 22(5), 323327.

Hamari, J. (2013). Transforming Homo Economicus into Homo Ludens: A Field Experiment on Gamification in a Utilitarian Peer-to-Peer trading Service. Electronic Commerce Research and Applications, 12(4), 236-245.

Hamari, J., Koivisto, J. \& Sarsa, H. (2014). Does Gamification Work?- A Literature Review of Empirical Studies on Gamification. In Proceedings of the $47^{\text {th }}$ Hawaii international conference on system sciences. IEEE, 3025-3034.

Hirschfeld, R. R. (2000). Does Revising the Intrinsic and Extrinsic Subscales of the Minnesota Satisfaction Questionnaire Short Form Make a Difference? Educational and Psychological Measurement, 60(2), 255-270.

Iosup, A. \& Epema, D. (2014). An Experience Report on Using Gamification in Technical Higher Education. In Proceedings of the $45^{\text {th }}$ ACM technical symposium on science education. ACM, 27-32.

Ijsselsteijn, W. A., Kort, Y. A. W. D. \& Poels, K. (2013). The Game Experience Questionnaire: Development of a self-report measure to assess the psychological impact of digital games. Manuscript in Preparation.

Jackson, S. A. \& Eklund, R. C. (2002). Assessing Flow in Physical Activity: The Flow State-2 and Dispositional Flow Scale-2. Journal of Sport \& Exercise Psychology, 24, 133-150.

Jung, J. H., Schneider, C., \& Valacich, J. (2010). Enhancing the motivational affordance of information systems: The effects of real-time performance feedback and goal setting in group collaboration environments. Management Science, 56(4), 724-742.

Lechler, T. (2001). Social Interaction: A Determinant of Entrepreneurial Team Venture Success. Small Business Economics, 16(4), 263-278.

Maan, J. (2013). Social Business Transformation through Gamification. International Journal of Managing Information Technology (IJMIT), 5(3).

Maslach, C. \& Leiter, M. P. (1997). The Truth About Burnout. San Francisco: Jossey-Bass. 
Mitgutsch, K. \& Alvaro, N. (2012). Purposeful by Design?: A Serious Game Design Assessment Framework. In Proceedings of the International Conference on the Foundations of Digital Games.

Moorman, R. H. \& Blakely, G. L. (1992). A preliminary report on a new measure of organizational citizenship behavior. In Proceedings of the annual meeting of the southern management association, 185-187.

Moorman, R. H. \& Blakely, G. L. (1995), Individualism-Collectivism as an Individual Difference Predictor of Organizational Citizenship Behavior. Journal of organizational behavior, 16(2), 127142.

Mowday, R. T., Porter, L. W. \& Steers, R. M. (1982). Employee-organization Linkages: The Psychology of Commitment, Absenteeism, and Turnover. New York, USA: Academic Press.

Nacke, L. E., Stellmach, S., Sasse, D. \& Lindley, C.A. (2009). Gameplay experience in a gaze interaction game. In Proceedings of the $5^{\text {th }}$ Conference on Communication by Gaze Interaction- COGAIN 2009:Gaze Interaction For Those Who Want It Most, The COGAIN Association, 49-54.

Nacke, L. E., Drachen, A. \& Göbel, S. (2010). Methods for Evaluating Gameplay Experience in Serious Gaming Context. International Journal of Computer Science in Sport, 9(2).

Raab-Steiner, E. \& Benesch, M. (2010). Der Fragebogen: Von der Forschungsfrage zur SPSS/PASWAuswertung. Wien: Facultas WUV Universitätsverlag.

Rauch, M. (2013). Best Practices for Using Enterprise Gamification to Engage Employees and Customers. In Kurosu, M. (Hrsg.): Human-Computer Interaction. Applications and Services. Heidelberg: Springer, S. 276-283.

Schaufeli, W. B., Salanova, M., González-Romá, V. \& Bakker, A. B. (2002). The Measurement of Engagement and Burnout: A Two Sample Confirmatory Factor Analytic Approach. Journal of Happiness Studies, 3(1), 71-92.

Schering, S. (2015).Mr. Gami Fication's Safe - An analysis tool for gamification in the business. Zugriff am 7.Mai 2015, unter http://interactivesystems.info/gamification-tool.

Ullrich, D. \& Diefenbach, S. (2010). From Magical Experience to Effortlessness: An Exploration of the Components of Intuitive Interaction. In Proceedings of the 6th nordic conference on humancomputer interaction: extending boundaries. ACM, 801-804.

Van Knippenberg, D. \& Sleebos, E. (2006). Organizational identification versus organizational commimtment: Self-definition, social exchange, and job attitudes. Journal of Organizational Behavior, 27(5), 571-584.

Weaver, G. R. \& Treviño, L. K. (1999). Compliance and Values Oriented Ethics Programs. Business Ethics Quarterly, 9(2), 315-335.

Werbach, K. \& Hunter, D. (2012). For the Win. How Game thinking can revolutionize your Business. Philadelphia, USA; Wharton Digital Press.

\section{Kontaktinformationen}

Katja Herrmanny

Interactive Systems, Universität Duisburg-Essen

Forsthausweg 2, 47057 Duisburg, Deutschland

katja.herrmanny@uni-due.de 
